\title{
Flexible high-performance graphene hybrid photodetectors functionalized with gold nanostars and perovskites
}

Yoon Ho Lee ${ }^{1}$, Seungyoung Park ${ }^{2,3}$, Yousang Won', Jungho Mun $\mathbb{0}^{4}$, Jae Hwan Ha, ${ }^{1,4}$, Jeong Hun Lee', Sang Hyuk Lee ${ }^{1}$, Jongmin Park', Jeonghee Yeom², Junsuk Rho (1) ${ }^{4,5}$, Hyunhyub Ko (1) ${ }^{2}$ and Joon Hak Oh (1)

\begin{abstract}
Hybrid materials in optoelectronic devices can provide synergistic effects that complementarily enhance the properties of each component. Here, flexible high-performance graphene hybrid photodetectors (PDs) are developed by introducing gold nanostars (GNSs) and perovskites for strong light trapping with hot electron transfer and efficient light harvesting characteristics, respectively. While pristine graphene PDs do not exhibit discernible photodetection properties due to the very low photon absorption and ultrafast charge carrier recombination, graphene PDs functionalized with GNSs and a densely covered perovskite layer exhibit outstanding photoresponsive properties with a photoresponsivity $(R)$ of $5.90 \times 10^{4} \mathrm{AW}^{-1}$ and a specific detectivity of $1.31 \times 10^{13}$ Jones, the highest values among those reported for perovskite-functionalized graphene PDs thus far. Moreover, we fabricated a flexible $10 \times 10$ PD array that shows well-resolved spatiotemporal mapping of light signals with excellent operational and mechanical stabilities at a bending radius down to $3 \mathrm{~mm}$ and in repeated bending tests for over 1000 cycles. Comprehensive analyses using finite-difference time-domain (FDTD) theoretical calculations, scanning near-field optical microscopy, and photoluminescence mapping reveal the effective light trapping effect of GNSs and the charge carrier transfer between the perovskite and graphene. This work provides a new design platform for flexible and high-performance photodetection systems.
\end{abstract}

\section{Introduction}

Graphene optoelectronics have attracted great attention for next-generation wearable devices due to their unique optical and electronic properties and the mechanical stability of graphene ${ }^{1-4}$. Graphene photodetectors (PDs) exhibit many desirable properties, such as a fast response arising from the high carrier mobility, mechanical flexibility, and ease of integration with other waveguide

\footnotetext{
Correspondence: Hyunhyub Ko (hyunhko@unist.ac.kr) or Joon Hak Oh (joonhoh@snu.ac.kr)

${ }^{1}$ School of Chemical and Biological Engineering, Institute of Chemical Processes, Seoul National University, 1 Gwanak-ro, Gwanak-gu, Seoul 08826 , Republic of Korea

${ }^{2}$ School of Energy and Chemical Engineering, Ulsan National Institute of Science and Technology (UNIST), Ulsan 44919, Republic of Korea Full list of author information is available at the end of the article These authors contributed equally: Yoon Ho Lee, Seungyoung Park
}

systems ${ }^{1,5-8}$. Currently, the need for a photodetection platform with high performance in terms of efficiency, wavelength range, flexibility, and integration is growing remarkably. The photoresponsivity $(R)$ of graphene-based PDs is mainly limited by the atomically thin nature of graphene with a low absorption cross-section ${ }^{9}$ and ultrafast charge carrier recombination ${ }^{1}$. Recently, several strategies to overcome this major drawback based on the development of graphene-based hybrid PD systems with plasmonic resonators ${ }^{10,11}$ or light-absorbing materials, such as quantum dots ${ }^{12,13}$, organic semiconductors ${ }^{14,15}$, or transition metal dichalcogenides ${ }^{16}$, have been suggested. However, the graphene PD performance must still be improved for practical applications.

In graphene PDs functionalized with plasmonic resonators, the $R$ can be enhanced by plasmonic effects.

\section{(c) The Author(s) 2020}

(c) (i) Open Access This article is licensed under a Creative Commons Attribution 4.0 International License, which permits use, sharing, adaptation, distribution and reproduction c. in any medium or format, as long as you give appropriate credit to the original author(s) and the source, provide a link to the Creative Commons license, and indicate if changes were made. The images or other third party material in this article are included in the article's Creative Commons license, unless indicated otherwise in a credit line to the material. If material is not included in the article's Creative Commons license and your intended use is not permitted by statutory regulation or exceeds the permitted use, you will need to obtain permission directly from the copyright holder. To view a copy of this license, visit http://creativecommons.org/licenses/by/4.0/. 
However, the photocurrent levels are on the order of tens to hundreds of nanoamperes, and the $R$ values are several milliamperes per watt $\left(\mathrm{mA} \mathrm{W}^{-1}\right)$ because single-atomthick graphene has almost no direct excitation of electron-hole pairs, resulting in an $R$ enhancement induced by hot electron transfer rather than by plasmonenhanced light scattering and electric fields ${ }^{10,17,18}$. Moreover, previous plasmonic resonators for graphene PDs use only a single type of plasmonic structure, such as metallic nanoparticles or nanopatterns ${ }^{10,11,19}$. Therefore, with the aim of boosting the performance of graphene PDs based on plasmonic resonators, it is essential to develop a system that can efficiently combine plasmoninduced light scattering with electric field enhancement effects by using new types of hybrid photosensing layers with more efficient plasmonic structures rather than using a single type of plasmonic structure.

In light-absorbing material-based graphene PDs, various light-absorbing materials that generate photoinduced charge carriers have been introduced for performance enhancement ${ }^{12,14,15}$. In particular, perovskite lightabsorbing materials with high optoelectronic performances have attracted great attention as next-generation optoelectronic light-absorbing materials owing to their superior photoelectrical properties ${ }^{6,20,21}$. However, further improvement of the device performance is still required for practical applications. Particularly, the formation of spatially inefficient perovskite islands on graphene creates perovskite grains with low crystallinity and decreases the photogenerated charge carrier density compared to compact perovskite layers on graphene, which is a major problem to be solved in graphene-perovskite hybrid systems ${ }^{6,21-23}$. Furthermore, in a dual hybrid system using both plasmonic nanoparticles and perovskite materials, the light scattering and electric field enhancement effects, which cannot enhance the photosensing performance in plasmonic nanoparticle-graphene single hybrid systems, are expected to greatly improve the light absorption of the perovskite materials, resulting in a large performance improvement. Previously, the dual hybrid system was explored by using inefficient and conventional sphere-type gold $(\mathrm{Au})$ nanoparticles with a noncompact (island type) perovskite layer, resulting in an insufficient improvement of the device performance due to the ineffective synergistic effect of the hybrid system ${ }^{22}$. Therefore, one major challenge for high-performance graphene hybrid PDs is to combine efficient plasmonic nanoparticles with a highly dense perovskite film in an effective manner. Herein, we describe a novel methodology for fabricating flexible high-performance graphene dual hybrid PDs composed of both plasmonic gold nanostars (GNSs) and perovskite materials (Fig. 1).

GNSs composed of a spherical core and several sharp tips show a highly efficient light trapping effect owing to

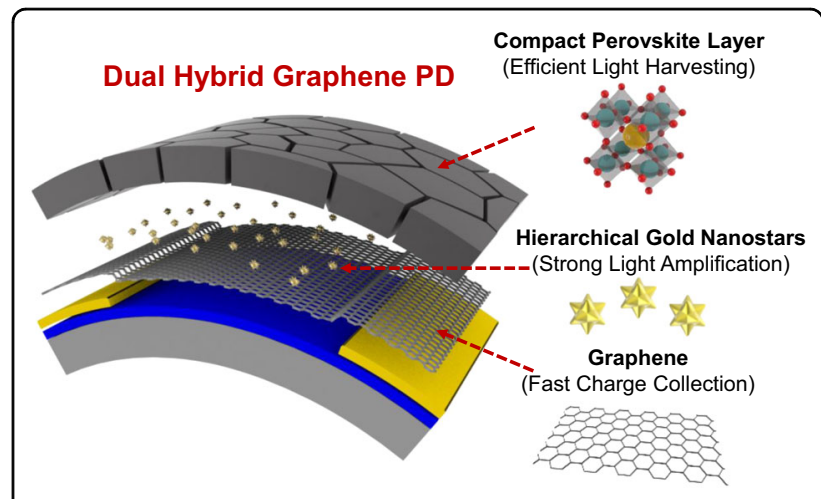

Fig. 1 Schematic illustration of a dual hybrid PD. Schematic of a graphene dual hybrid PD composed of a perovskite material and GNSs with illustration of the key role of each component in the synergistic effect for optical sensing.

the very strong local electric fields generated by the hybridization of tip and core plasmon modes ${ }^{24}$. Flexible graphene/GNS/perovskite PDs (G-GNS-P-PDs) exhibit a significantly enhanced $R$ of $5.90 \times 10^{4} \mathrm{~A} \mathrm{~W}^{-1}$ and specific detectivity $\left(D^{\prime \prime}\right)$ of $1.31 \times 10^{13}$ Jones, which are 7 -fold and 2-fold higher than those of graphene/perovskite PDs (GP-PDs) and 7.7 million-fold and 100-fold greater than those of pristine perovskite PDs (P-PDs), respectively. These $R$ and $D^{\prime \prime}$ are the highest among those reported for methylammonium lead iodide $\left(\mathrm{MAPbI}_{3}\right)$ perovskite-based graphene PDs thus far. Furthermore, flexible $10 \times 10 \mathrm{G}$ GNS-P-PD arrays prepared with a flexible plastic substrate could detect incoming photonic signals at a high resolution with excellent mechanical stability under a bending radius down to $3 \mathrm{~mm}$ and in repeated bending tests over 1000 cycles. Theoretical calculations, scanning near-field optical microscopy (SNOM) analysis, and photoluminescence (PL) mapping analysis were also performed to substantiate the strong plasmonic effects of the GNSs and the efficient charge carrier transfer between the perovskite and graphene. The graphene hybrid PDs described herein are expected to contribute to the development of a wide range of optoelectronic devices, including perovskite-based photovoltaics, light-emitting diodes, lasers, and organic and quantum dot-based optoelectronic devices.

\section{Materials and methods}

\section{Synthesis and characterizations of GNSs}

The GNSs consist of a spherical core surrounded by multibranched sharp tips. The electromagnetic field is strongly concentrated at the end of the sharp tips via a lightning-rod effect. The GNSs were synthesized by a surfactantless method using $75 \mathrm{~mL}$ of 2-[4-(2-hydroxyethyl)-1-piperazinyl] ethane-sulfonic acid (HEPES) as a shape control agent to control the tip-shaped branches 
of the GNSs during the reduction process. The spherical $\mathrm{Au}$ seed particles of $18 \mathrm{~nm}$ diameter were enlarged by the reduction of $\mathrm{Au}$ ions to Au with $360 \mu \mathrm{L}$ of $100 \mathrm{mM}$ hydroxylamine $\left(\mathrm{NH}_{2} \mathrm{OH}\right)$ to form the multibranched GNSs, followed by dropwise addition of $15 \mathrm{~mL}$ of $\mathrm{HAuCl}_{4}$ solution $(0.8 \mathrm{mM})$ in HEPES solution. All synthetic processes were carried out at $4{ }^{\circ} \mathrm{C}$. The final GNS solution was concentrated 90 times by centrifugation at $6000 \mathrm{rpm}$ for $30 \mathrm{~min}$ and then stored at $4{ }^{\circ} \mathrm{C}$ until use. The morphology of GNS particles was investigated by field emission scanning electron microscopy (SEM) (Nano 230, FEI) and transmission electron microscopy (TEM) (JEM-2100, JEOL). The optical properties were characterized by UV-Vis spectrophotometry (V-670, JASCO).

\section{Fabrication and characterization of photodetectors}

$\mathrm{Cr}(4 \mathrm{~nm}) / \mathrm{Au} \quad(30 \mathrm{~nm})$ electrodes were thermally evaporated through a shadow mask onto a noctadecyltrichlorosilane (OTS)-treated $\mathrm{SiO}_{2} / \mathrm{Si}$ substrate (width: $50 \mu \mathrm{m}$, length: $1000 \mu \mu \mathrm{m}$ ). The thickness of the $\mathrm{SiO}_{2}$ was $300 \mathrm{~nm}$. A graphene film was grown on copper foil by the conventional chemical vapor deposition (CVD) method using $\mathrm{H}_{2}$ and $\mathrm{CH}_{4}$ gas. After the growth of graphene, poly(methyl methacrylate) (PMMA) was coated onto the graphene surface. Subsequently, the copper foil under the graphene film was etched using ammonium persulfate $\left[\left(\mathrm{NH}_{4}\right)_{2} \mathrm{~S}_{2} \mathrm{O}_{8}\right]$ aqueous solution $(0.1 \mathrm{M})$. Then, the PMMA/graphene was wet-transferred onto the $\mathrm{Si} /$ $\mathrm{SiO}_{2}$ substrate and annealed at $130^{\circ} \mathrm{C}$ for $30 \mathrm{~min}$. The PMMA-supporting film was removed with acetone. Then, ethanol and a $4 \times$ dilution of the GNS solution were sequentially dropped and spin coated onto the film after $1 \mathrm{~h} . \mathrm{PbI}_{2}$ (Aldrich) was thermally evaporated with a mask. Then, methylammonium iodide (MAI) (Solarmer) solution (40 mg in isopropanol) was spin coated onto the film at $2000 \mathrm{rpm}$ for $40 \mathrm{~s}$, followed by annealing at $135^{\circ} \mathrm{C}$ for $20 \mathrm{~min}$. A thin film of parylene $\mathrm{C}$ was deposited by a CVD process. A parylene deposition system (Obang Technology Co., Ltd) was used for the parylene $\mathrm{C}$ deposition. The system consists of three parts: a vaporizer, a pyrolyzer, and a deposition chamber. In addition, the CVD process was primarily composed of three steps: vaporization, pyrolysis, and polymerization. First, the system was vacuumed to below $12 \mathrm{~m}$ Torr. Next, the pyrolyzer was heated to $690^{\circ} \mathrm{C}$, and the vaporizer was heated to $180^{\circ} \mathrm{C}$. After the heating process, the parylene $\mathrm{C}$ powder was first vaporized from the solid form and then pyrolyzed from the parylene $\mathrm{C}$ dimer into a monomer. Finally, the monomer gas flowed up to the deposition chamber and deposited a transparent polymer thin film onto the surfaces of the sample. During the final step, the pressure in the deposition chamber was kept at $\sim 20$ mTorr for a couple of hours until the process finished. According to our deposition conditions, a $\sim 1-\mu \mathrm{m}$-thick thin film was formed by using $1.8 \mathrm{~g}$ of parylene $\mathrm{C}$. The $I-V$ characteristics of the PDs were measured under vacuum conditions using a Keithley 4200-SCS semiconductor parametric analyzer. Raman spectra were taken with a $\mathrm{He}-\mathrm{Ne}$ laser $(532 \mathrm{~nm})$ as the excitation source using confocal Raman microscopy (Alpha 300S, WITec, Germany). The crystalline structures were examined by $\mathrm{X}$-ray diffraction (XRD) (Bruker AXS, D8 Advance). UV-Vis absorption spectra were measured on a spectrophotometer (Carry 5000). SEM images were obtained using a Hitachi cold SEM microscope. The PL spectra and integrated intensity maps were obtained by a confocal Raman microscopy system with measurements conducted at room temperature (Alpha 300s, WiTec, Germany). The wavelength and power of the laser were $532 \mathrm{~nm}$ and $0.3 \mathrm{~mW}$, respectively. The grating and integration times of the spectrometer were $600 \mathrm{~g} / \mathrm{mm}$ and $0.5 \mathrm{~s}$, respectively.

\section{Fabrication and characterization of flexible photodetectors and arrays}

$\mathrm{Cr}(4 \mathrm{~nm}) / \mathrm{Au}(30 \mathrm{~nm})$ electrodes were thermally evaporated through a shadow mask on a polyethylene terephthalate (PET) substrate (width: $50 \mu \mathrm{m}$, length: $1000 \mu \mathrm{m}$ for flexibility test; width: $30 \mu \mathrm{m}$, length: $750 \mu \mathrm{m}$ for array test). The thickness of the $\mathrm{SiO}_{2}$ was $300 \mathrm{~nm}$. CVD-grown graphene was transferred onto the substrate. Then, ethanol and the GNS solution were sequentially dropped and spin coated onto the graphene after $1 \mathrm{~h} . \mathrm{PbI}_{2}$ (Aldrich) was thermally evaporated with a mask. Subsequently, MAI solution ( $40 \mathrm{mg}$ in isopropanol) was spin coated onto the film at $2000 \mathrm{rpm}$ for $40 \mathrm{~s}$, followed by annealing at $110^{\circ} \mathrm{C}$ for $30 \mathrm{~min}$. The $I-V$ characteristics of the PDs were measured under vacuum conditions using a Keithley 4200-SCS semiconductor parametric analyzer.

\section{Measurement of the complex refractive index of perovskites}

The complex refractive index of the semiconductor was measured with an ellipsometer (J.A. Woollam). The perovskite layer was prepared using the same method as for device fabrication on $\mathrm{SiO}_{2} / \mathrm{Si}$ wafer (4-nm-thick $\mathrm{SiO}_{2}$ ).

\section{SNOM measurement}

SNOM images were obtained using a confocal SNOM/ atomic force microscope (Alpha 300, WITec). Selectively GNS-coated $\mathrm{Si} / \mathrm{SiO}_{2}$ substrates were scanned in the SNOM contact reflection mode with a set point of $1 \mathrm{~V}$. The scanning size and velocity for the SNOM image were $20 \times 20 \mu \mathrm{m}$ and $20 \mu \mathrm{m} \mathrm{s}^{-1}$, respectively. A 532-nm laser wavelength was coupled to the subwavelength aperture (60 $\mathrm{nm}$ in diameter) to generate the optical near field on metal surfaces. The scattered light from the sample was collected by a microscope objective $(0.4 \mathrm{NA}, 20 \times)$. 
Finite-difference time-domain (FDTD) simulation methods

The absorbance and electric field density were calculated using a commercial FDTD solver (Lumerical). The simulation setup had periodic boundary conditions in the $x$ - and $y$-directions with a periodicity of $1 \mu \mathrm{m}$ and a perfectly matched layer in the $z$-direction. The planewave source was excited, and the maximum mesh size near the GNS was $0.5 \mathrm{~nm}$. The thickness of the perovskite active layer was $160 \mathrm{~nm}$. The optical constants of Au were taken from tabulated data (Johnson and Christy) and those of the perovskite from ellipsometry measurements.

\section{Results and discussion}

\section{Synthesis and characterization}

GNSs were synthesized by a surfactantless synthetic approach based on enlargement of spherical Au nanoparticles by reduction of $\mathrm{Au}$ ions to $\mathrm{Au}$ with the aid of hydroxylamine $\left(\mathrm{NH}_{2} \mathrm{OH}\right)^{25}$. The concentration of the asprepared GNS solution was $\sim 1.3 \times 10^{-11} \mathrm{M}$ from the calculation based on the UV/Vis spectra. The GNS solution was concentrated 90 times by centrifugation and then stored at $4{ }^{\circ} \mathrm{C}$ until use $e^{26}$. The morphology of the GNSs was investigated by TEM analysis to investigate the multibranched tips of the GNSs. Figure 2a shows TEM images of the GNSs, indicating their uniformity with a spherical core diameter of $55 \pm 7 \mathrm{~nm}$ and a sharp tip size of $12 \pm 3 \mathrm{~nm}$. The right panel in Fig. 2a shows an enlarged image of the tip of a GNS. The fringe spacing at the tip of the GNS is $0.24 \mathrm{~nm}$, which corresponds to the (111) lattice plane of face-centered cubic gold. The inset in the right panel shows the corresponding fast Fourier transform image, which indicates a $d$-spacing of $0.24 \mathrm{~nm}$ along the (111) axis. The UV-Vis absorption spectra of the GNSs show a strong absorption band at $\sim 668 \mathrm{~nm}$, which is attributed to the localized surface plasmon resonance (LSPR) of the sharp tips of the GNSs (Fig. 2b). The additional shoulder peak at $\sim 533 \mathrm{~nm}$ is attributed to the plasmon mode of the spherical core. Single-layer graphene was synthesized on copper foil via CVD and then transferred onto a substrate ${ }^{27}$. As shown in Fig. 2c, the uniformity and high quality of the single-layer graphene are well demonstrated by the TEM and selectedarea diffraction (SAED) observations. The Raman spectrum also shows the typical features of graphene grown by thermal decomposition: the $2 D$ peak at $2750 \mathrm{~cm}^{-1}$ and the $G$ peak at $1584 \mathrm{~cm}^{-1}$. The minimal intensity of the defect-related $D$ peak at $\sim 1325 \mathrm{~cm}^{-1}$ indicates the good quality of the graphene film. The Raman spectra of the graphene layers exhibit $2 D$ peaks with twice the intensity of the $G$ peaks, indicating a single-layer graphene structure, as shown in Fig. 2 d.

The fabrication process of G-GNS-P-PDs is illustrated in Fig. 3a. Gold electrodes were thermally deposited on an OTS-treated $\mathrm{SiO}_{2} / \mathrm{Si}$ substrate. Then, CVD-grown graphene on copper $(\mathrm{Cu})$ foil was transferred onto the substrate. Following this process, the graphene layer was coated with GNSs by dropping a solution containing ethanol and GNSs dispersed in distilled water onto the layer for $30 \mathrm{~min}$, followed by blow-drying with $\mathrm{N}_{2}$ gas. Subsequently, lead iodide $\left(\mathrm{PbI}_{2}\right)$ was thermally deposited onto the device followed by spin coating with MAI solution. Finally, a 180-nm-thick perovskite was crystallized onto the device by annealing, and Au electrodes were thermally evaporated onto the film with a mask. For comparison, graphene/GNS (G-GNS), perovskite-only, GNS/perovskite (GNS-P), and graphene/perovskite (G-P) films were also prepared. Figure $3 \mathrm{~b}$ shows an SEM image of the well-dispersed GNSs on the graphene film. The morphologies of the perovskite-only, GNS-P, G-P, and GGNS-P films are shown in Supplementary Fig. S1a-c and Fig. 3c. The surface morphologies of the G-P and G-GNS$P$ films show relatively high surface coverage and smooth perovskite film compared to the surface morphologies of the perovskite-only and GNS-P films without graphene, presumably due to the facilitated infiltration of the perovskite precursor $^{28}$. The cross-sectional SEM image of the G-GNS-P film in Supplementary Fig. S2 also displays smooth and relatively high surface coverage of the perovskite film. Furthermore, the embedded GNSs under the perovskite layer, which efficiently introduce plasmonic effects, including hot electron transfer and light trapping, without incident light blocking by the perovskite light-absorbing layer, are clearly observed in the enlarged cross-sectional SEM image in Fig. 3d. Figure 3e shows the XRD patterns obtained from each type of photoactive layer (except for G-GNSs) on the OTStreated $\mathrm{SiO}_{2} / \mathrm{Si}$ substrates, all of which exhibit wellmatched perovskite crystalline structures ${ }^{29}$. Figure $3 \mathrm{f}$ shows the UV-Vis absorption spectra of the pristine perovskite layer, GNS-P layer, G-P layer, and G-GNS-P layer. The absorption in the visible light region increased with the introduction of the perovskite and GNSs into the graphene due to the high light absorption properties of the perovskite and the plasmonic light trapping effect of the GNSs. Therefore, the G-GNS-P layer showed the highest light absorption.

\section{Photodetector performance}

Graphene PDs decorated with GNSs only (G-GNS-PDs) without perovskite light absorption materials showed poor photosensing performances with a low photocurrent of $0.08 \mathrm{~mA}$ under high intensity polychromatic light $\left(\lambda=450-650 \mathrm{~nm}\right.$, intensity $\left.=36 \mathrm{~mW} \mathrm{~cm}^{-2}\right)$, as shown in Supplementary Fig. S3. Therefore, we focused on the PD performance analysis of G-P-PDs and G-GNS-P-PDs. Moreover, P-PDs and P-GNS-PDs were also fabricated and analyzed to investigate the performance enhancement characteristics due to the introduction of GNSs into 
$\mathbf{a}$

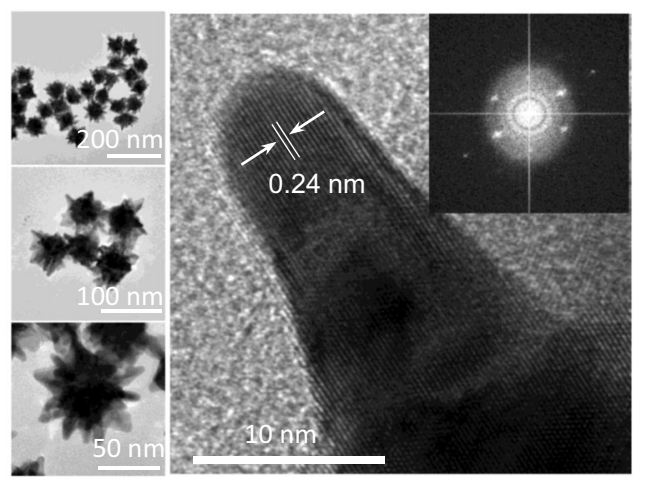

c

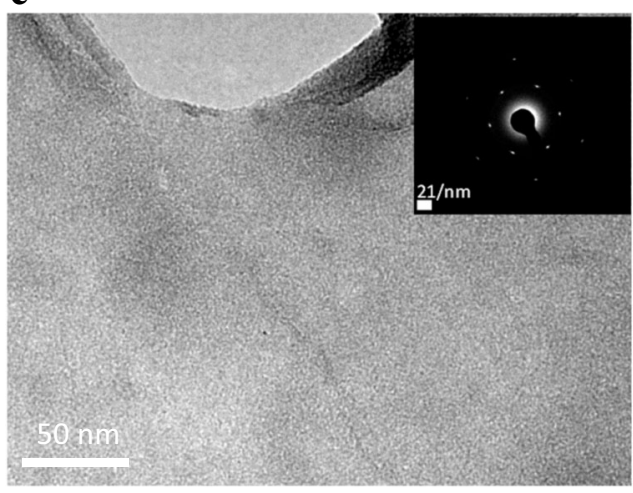

b

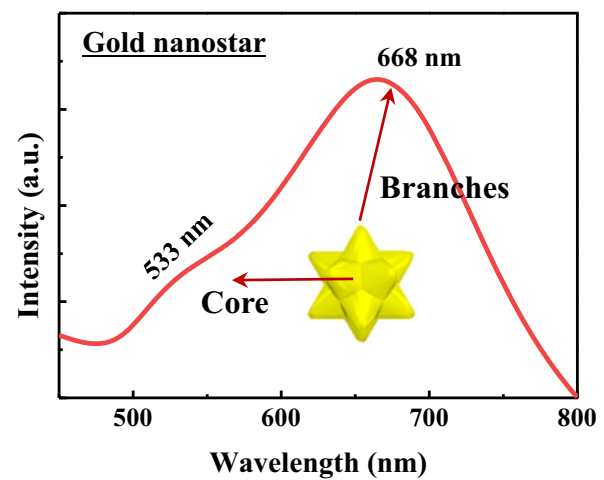

d

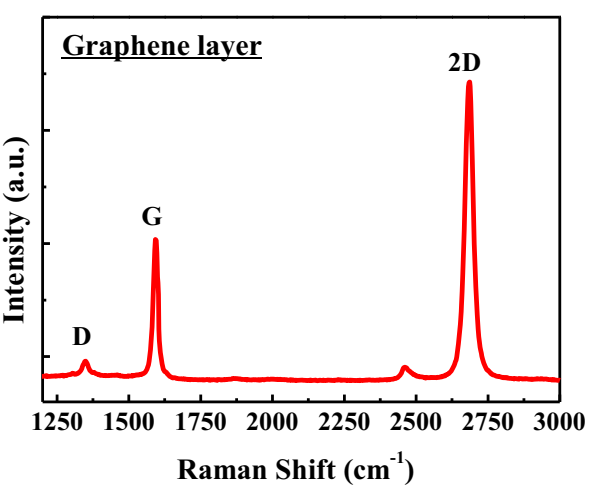

Fig. 2 Material characterization. a TEM images of GNSs. The inset image in the right image shows the corresponding fast Fourier transform image, which indicates a $d$-spacing of $0.24 \mathrm{~nm}$ along the (111) axis. b Absorbance spectra of dispersed GNSs in DI water. c TEM image of single-layer CVDgrown graphene, and SAED pattern from the TEM image of the graphene (inset). $\mathbf{d}$ Raman spectra of graphene.

graphene-free pristine perovskite-based PD systems. Figure $4 \mathrm{a}$ schematically illustrates the device structures, including those of the P-PDs, GNS-P-PDs, G-P-PDs, and G-GNS-P-PDs. A schematic image of the G-GNSPDs is shown in Supplementary Fig. S4. A photograph showing the measurement of the device characteristics is shown in Supplementary Fig. S5. The photosensitivity of the G-GNS-P-PDs was estimated by measuring the current-voltage $(I-V)$ characteristics under blue $(460 \mathrm{~nm})$, green $(532 \mathrm{~nm})$, and red $(670 \mathrm{~nm})$ light with various light intensities. Supplementary Fig. S6 shows the $I-V$ characteristics of the P-PDs, GNS-P-PDs, G-P-PDs, and GGNS-P-PDs under blue light. The current increased gradually as the illumination power increased due to the increased amount of incident photons ${ }^{30,31}$. Particularly, as shown in Fig. 4b, the photocurrent levels were greatly increased upon utilization of the graphene and perovskite hybrid system due to the high mobility of graphene and the remarkable photoresponsivity of the perovskite materials. Furthermore, the introduction of GNSs further increased the photocurrents owing to the light trapping effects of the plasmonic GNSs. These results indicate that both the GNSs and perovskite materials were effectively introduced to improve the performance of graphene PDs.

To investigate the photocurrent response depending on the incident optical power, the $R$ and external quantum efficiency (EQE) of the device were calculated using the following equations:

$$
\begin{aligned}
& R=\frac{I_{\mathrm{ph}}}{P_{\text {inc }}}=\frac{I_{\text {light }}-I_{\text {dark }}}{P_{\text {inc }}}, \\
& \mathrm{EQE}=\frac{\left(I_{\text {light }}-I_{\text {dark }}\right) h c}{e P_{\text {int }} A \lambda},
\end{aligned}
$$

where $I_{\mathrm{ph}}$ is the photocurrent, $I_{\text {light }}$ is the current under illumination, $I_{\text {dark }}$ is the dark current, $P_{\text {inc }}$ is the incident illumination power on the effective area, $P_{\text {int }}$ is the incident light intensity (i.e., the incident optical power density), $h$ is the Planck constant, $c$ is the speed of light, $e$ is the electron charge, $A$ is the active area, and $\lambda$ is the wavelength. In addition, $D^{*}$ is also one of the key merits of a PD and usually describes the smallest detectable signal 
$\mathbf{a}$
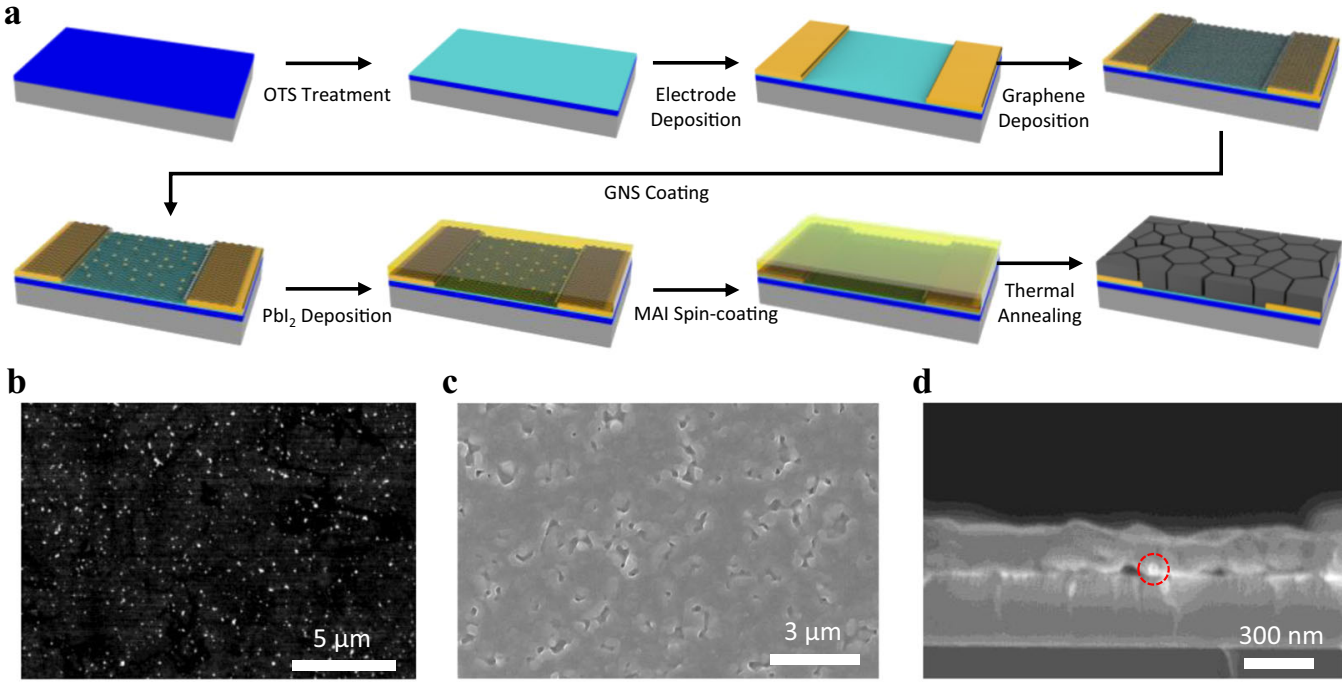

S Coating

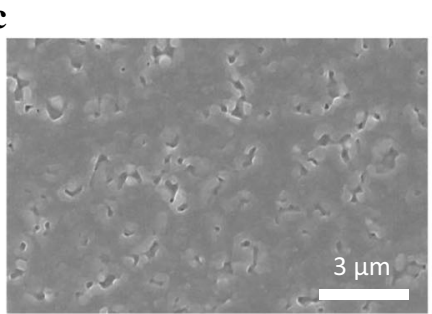

d

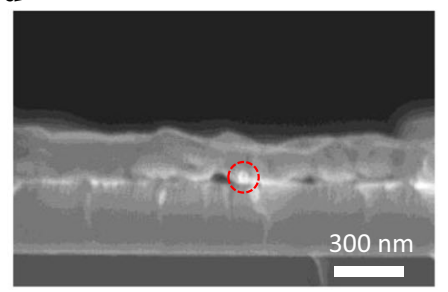

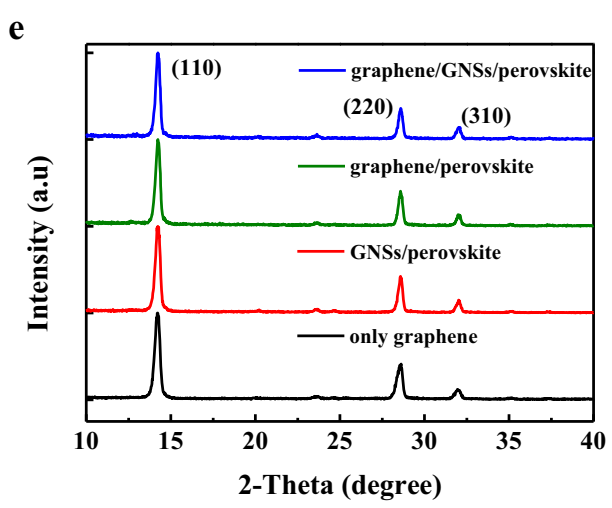

e f

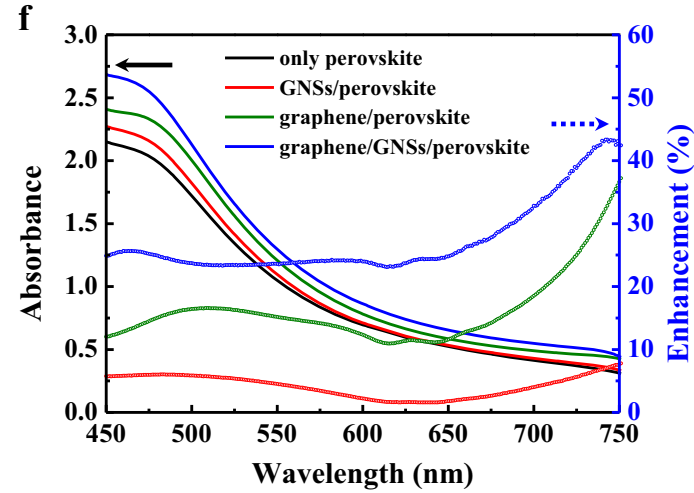

Fig. 3 Device fabrication and characterization. a Schematic diagrams of the fabrication process for G-GNS-P-PDs. b SEM image of the GNSdispersed graphene layer. c SEM image of the G-GNS-P layer. $\mathbf{d}$ Cross-sectional SEM image of the G-GNS-P layer (a GNS is indicated by a circle with a red dotted line). e XRD pattern and $\mathbf{f}$ UV-Vis absorption spectra of photoactive layers (only-perovskite, GNS-P, G-P, and G-GNS-P).

(the following equations):

$$
\begin{aligned}
& D^{*}=\frac{(\mathrm{AB})^{\frac{1}{2}}}{\mathrm{NEP}}\left(\mathrm{cm} \mathrm{Hz}^{\frac{1}{2}} \mathrm{~W}^{-1}\right), \\
& \mathrm{NEP}=\frac{\bar{i}_{n}^{2}}{R}(\mathrm{~W}),
\end{aligned}
$$

where $B$ is the bandwidth, NEP is the noise equivalent power, and $\bar{i}_{n}^{1 / 2}$ is the measured noise current. If the shot noise from the dark current is the major contributor to the noise limiting the detectivity, then the detectivity can be simplified as followed:

$$
D^{*}=\frac{R}{\left(2 \mathrm{e} \times I_{\mathrm{dark}} / A\right)^{1 / 2}} .
$$

The precise $D^{*}$ values calculated by measuring the real NEP values taking into account all noise currents according to Eq. (3) may be lower than the value calculated through Eq. (5), as discussed by Fang et al. ${ }^{32}$. However, due to the limitations of the equipment, we calculated the $D^{*}$ values using Eq. (5). The key parameters of PDs with different patterns are summarized in Table 1. The $R, \mathrm{EQE}$, and $D^{*}$ values of different types of PDs as a function of the illuminated light power under blue light are shown in Fig. 4c-e, respectively. The enhancements in $R$, EQE, and $D^{*}$ significantly increased as the light intensity decreased, which is a commonly observed phenomenon $^{30,31}$. Under a light intensity of $10 \mu \mathrm{W} \mathrm{cm} \mathrm{cm}^{-2}, R$ was improved significantly by up to $7.3 \times 10^{6}$-fold, from $0.008 \mathrm{~A} \mathrm{~W}^{-1}$ for the P-PDs to $58952.098 \mathrm{~A} \mathrm{~W}^{-1}$ for the G-GNS-P-PDs, while those of the GNS-P-PDs and G-PPDs were 0.013 and $8400.017 \mathrm{~A} \mathrm{~W}^{-1}$, respectively. This suggests that the enhancement in the photocurrent of the G-GNS-P-PDs was greater than those of the GNS-P-PDs and G-P-PDs due to the synergistic effect of utilizing both graphene and GNSs. EQE was also improved significantly 
a

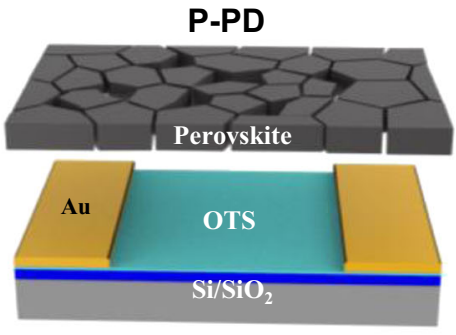

GNS-P-PD
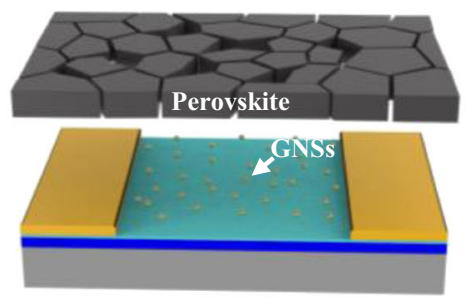

c

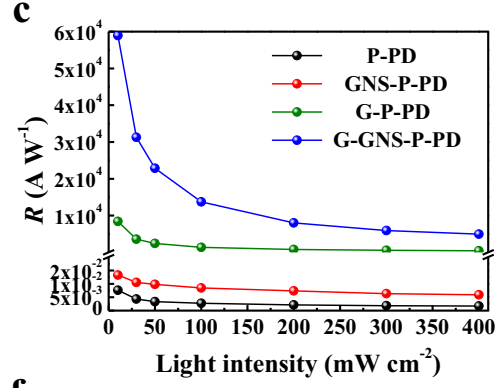

f

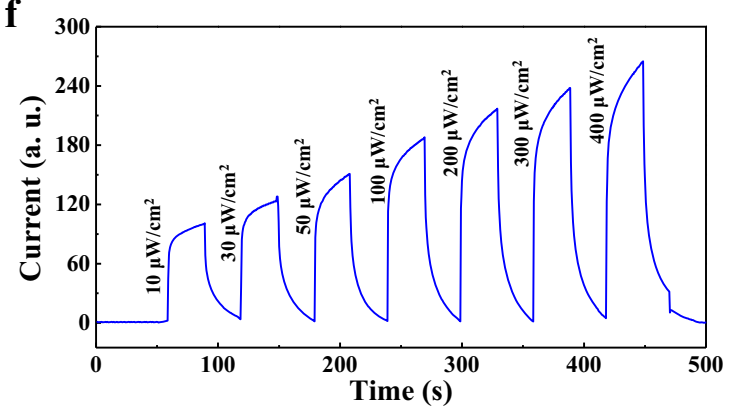

G-P-PD

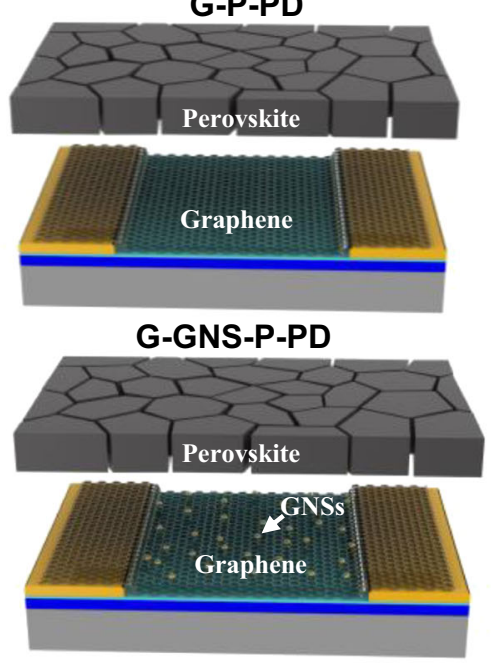

d

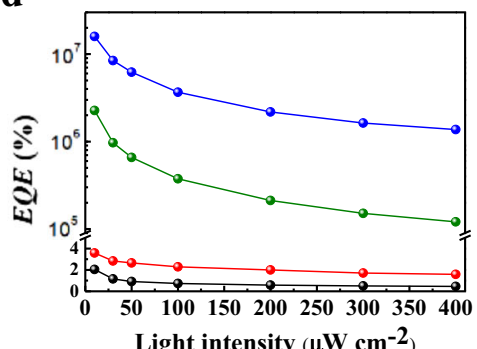

b

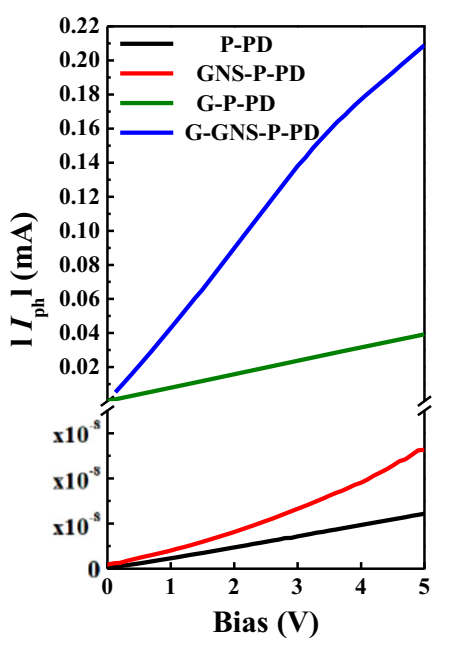

e

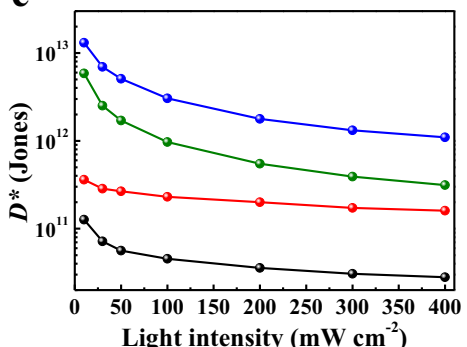

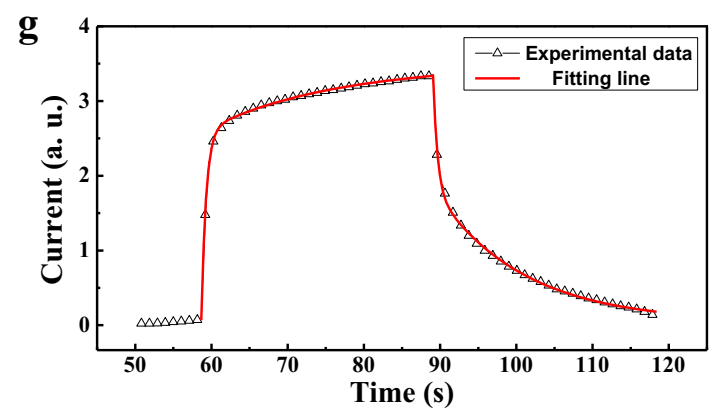

Fig. 4 Evaluation of device performances. a Schematic diagrams of the device structures for P-PDs, GNS-P-PDs, G-P-PDs, and G-GNS-P-PDs. $\mathbf{b}$ /ph-V characteristics of P-PDs, GNS-P-PDs, G-P-PDs, and G-GNS-P-PDs under $10 \mu \mathrm{W} \mathrm{cm} \mathrm{cm}^{-2}$ of blue light (460 nm). c $R$, d EQE, and e D* of P-PDs, GNS-P-PDs, G-P-PDs, and G-GNS-P-PDs and $\mathbf{f}$ photoswitching characteristics of G-GNS-P-PDs under various intensities of blue light (-5 $\mathrm{V}$ bias). $\mathbf{g}$ Rising and decay time fitting curve of G-GNS-P-PDs under blue light illumination $\left(10 \mu \mathrm{W} \mathrm{cm}^{-2}\right)$.

from $2 \%$ for the P-PDs to $1.59 \times 10^{7} \%$ for the G-GNS-PPDs, while those of the GNS-P-PDs and G-P-PDs were $4 \%$ and $1.79 \times 10^{6} \%$, respectively, showing photomultiplication with a trend similar to that of $R$. Moreover, $D$ " was enhanced from $1.3 \times 10^{11}$ Jones for the P-PDs to $130.9 \times$ $10^{11}$ Jones for the G-GNS-P-PDs, while those of the GNSP-PDs and G-P-PDs were $3.6 \times 10^{11}$ Jones and $58.7 \times 10^{11}$ Jones, respectively. Notably, the $D^{\prime \prime}$ values of the G-GNSP-PDs and G-P-PDs showed relatively low enhancements compared to the $R$ values because of the highly enhanced $I_{\text {dark }}$ of these devices. Other device characteristics under green and red light exhibiting identical tendencies to those under blue light are shown in Supplementary Figs. S7 and S8, respectively.

The dependence of $R$ on the light intensity $(P)$ can be fitted with power functions, as shown in Supplementary Fig. S9a. One of the reasons for the reduced responsivity with regard to the illumination intensity is the increased probability of carrier recombination as the carrier density increases $^{31,33}$. As illustrated in Supplementary Fig. S9b, most photoinduced carriers in the G-GNS-P-PDs are generated at the interface between graphene and the 
Table 1 Device characteristics of PDs (at $-5 \mathrm{~V}$ bias under $10 \mu \mathrm{W} \mathrm{cm}{ }^{-2}$ ).

\begin{tabular}{lrrr}
\hline PD & $\boldsymbol{R}\left(\mathbf{A ~ W}^{-\mathbf{1}}\right)$ & \multicolumn{1}{c}{ EQE (\%) } & $\boldsymbol{D}^{*}$ (Jones) \\
\hline Under blue light & & & \\
P-PDs & 0.008 & 2.0 & $1.3 \mathrm{E}+11$ \\
GNS-P-PDs & 0.013 & 3.6 & $3.6 \mathrm{E}+11$ \\
G-P-PDs & 8400.017 & $2,265,857.2$ & $58.7 \mathrm{E}+11$ \\
G-GNS-P-PDs & $58,952.098$ & $15,901,994.9$ & $130.9 \mathrm{E}+11$ \\
Under green light & & & \\
P-PDs & 0.008 & 2.0 & $1.4 \mathrm{E}+11$ \\
GNS-P-PDs & 0.023 & 5.4 & $6.3 \mathrm{E}+11$ \\
G-P-PDs & 7951.446 & $1,854,576.1$ & $55.5 \mathrm{E}+11$ \\
G-GNS-P-PDs & $54,065.759$ & $12,610,167.2$ & $119.9 \mathrm{E}+11$ \\
Under red light & & & \\
P-PDs & & 1.4 & $1.3 \mathrm{E}+11$ \\
GNS-P-PDs & 0.008 & 2.8 & $4.1 \mathrm{E}+11$ \\
G-P-PDs & 8042.157 & $1,489,388.2$ & $56.2 \mathrm{E}+11$ \\
G-GNS-P-PDs & $53,748.488$ & $9,954,091.5$ & $119.3 \mathrm{E}+11$ \\
\hline
\end{tabular}

perovskite due to the near-field enhancement of the GNSs. The probability for charge carrier recombination is greatly reduced because most of the charge carriers are located close to the perovskite-graphene interface. This contributes to the observed smaller decline of $R$ upon increasing light intensity in the G-GNS-P-PDs. Notably, the $R$ of our G-GNS-P-PDs $\left(58,952.098 \mathrm{~A} \mathrm{~W}^{-1}\right)$ is 28 times greater than the $R$ of a previously reported graphene hybrid $\mathrm{PD}^{22}$ that used both spherical plasmonic $\mathrm{Au}$ nanoparticles and a perovskite material. The $R$ and $D^{\prime \prime}$ of our G-GNS-P-PD were also compared to those of other G-P $\mathrm{MAPbI}_{3}$-based PDs in a two-electrode system, as shown in Supplementary Table S1 and Supplementary Fig. S10. The $R$ and $D^{*}$ values are the highest among those reported for $\mathrm{MAPbI}_{3}$ perovskite-based graphene PDs thus far. This means that the perovskite material and plasmonic GNSs were very efficiently introduced into graphene in our hybrid PD system.

To optimize the light sensing performance, the dependences of the G-GNS-P-PD and GNS-P-PD performances on the degree of GNS dispersion on the substrate were also studied by controlling the concentration of the GNS solution. As the concentration of the $\mathrm{Au}$ solution decreased, the density of GNSs on the substrate decreased, as shown in Supplementary Fig. S11, and the performance of the device also decreased, as shown in Supplementary Fig. S12. Figure $4 \mathrm{f}$ shows the on/off photoswitching characteristics of the sensors under blue light with various intensities. The devices maintained stable output signals under repeated on/off cycles and exhibited increased current with increasing light intensity. Upon illumination, the current of the device rises within $2 \mathrm{~s}$. The rapid photocurrent rise is followed by a slower increase in the photocurrent for $\sim 28 \mathrm{~s}$ before saturation. After the light is turned off, the photocurrent drops rapidly within $1 \mathrm{~s}$, followed by a slow decay process for more than $29 \mathrm{~s}$ before it returns to its initial current in the dark condition. The rise time $\left(t_{r}\right.$, defined as the time to rise from 0 to $80 \%$ of the maximum photocurrent) and decay time $\left(t_{d}\right.$, defined as the time to fall from 100 to $20 \%$ of the maximum photocurrent) were measured as 2.5 and $11.9 \mathrm{~s}$, respectively, as shown in Fig. 4g. Other photoswitching characteristics under green and blue light are shown in Supplementary Fig. S13. Moreover, the moisture stabilities of the G-GNS-P-PDs with and without the parylene encapsulation layer were tested in air at room temperature with a relative humidity of $\sim 60 \%$. As shown in Supplementary Fig. S14, the PDs without the parylene encapsulation layer of the device with the pure perovskite film first exhibited a fast degradation rate, retaining only $<30 \%$ of the initial $R$ after $9 \mathrm{~h}$. In contrast, the PDs with the parylene encapsulation layer retained over $95 \%$ of their original $R$ after $350 \mathrm{~h}$ under the same conditions.

\section{Investigation of the graphene/GNS/perovskite hybrid system}

The effects of graphene and GNSs on the enhanced performance of G-GNS-P-PD devices were investigated (Fig. 5). Figure 5a shows the steady-state PL spectra of the perovskite-only, GNS-P, G-P, and G-GNS-P films. These films were prepared on OTS-treated $\mathrm{SiO}_{2} / \mathrm{Si}$ substrates. The PL intensity of the G-P film was significantly decreased compared to that of the perovskite-only film. This PL quenching resulted from effective charge carrier transfer from graphene to the perovskite film or from the perovskite film to graphene ${ }^{6,20}$. The charge carrier transfer occurred effectively through the electronic coupling between graphene, with a widely distributed $\pi$ orbital, and the perovskite film ${ }^{34}$. In the presence of a graphene layer, the electrons of graphene are transferred to the valence band of the perovskite, which causes a limited density of states for recombination of photoexcited electron-hole pairs in the perovskite, resulting in higher sustainability of photoexcited electrons in the conduction band of the perovskite. This mechanism results in the dramatic quenching of the PL intensity in the G-P film.

The graphene/GNS/perovskite films showed enhanced PL intensities due to the significant near-field effect induced by LSPR coupling of the GNSs, resulting in an overall increase in the number of photogenerated electrons compared to the films with graphene and perovskite layers $^{35,36}$. This enhanced PL intensity was similarly 
a

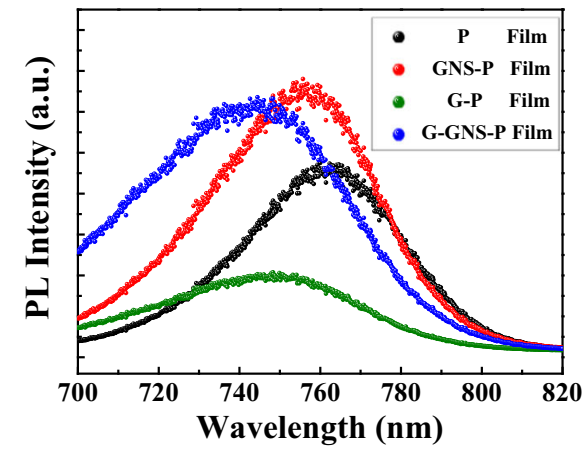

c

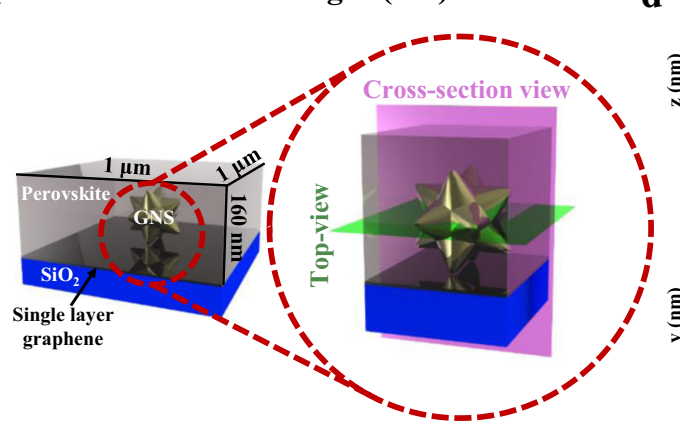

e

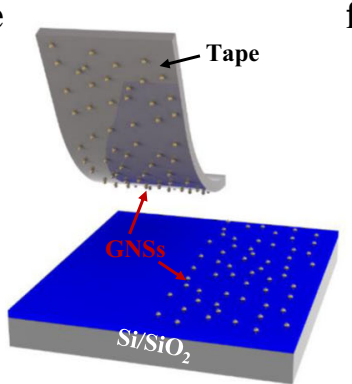

d

f b
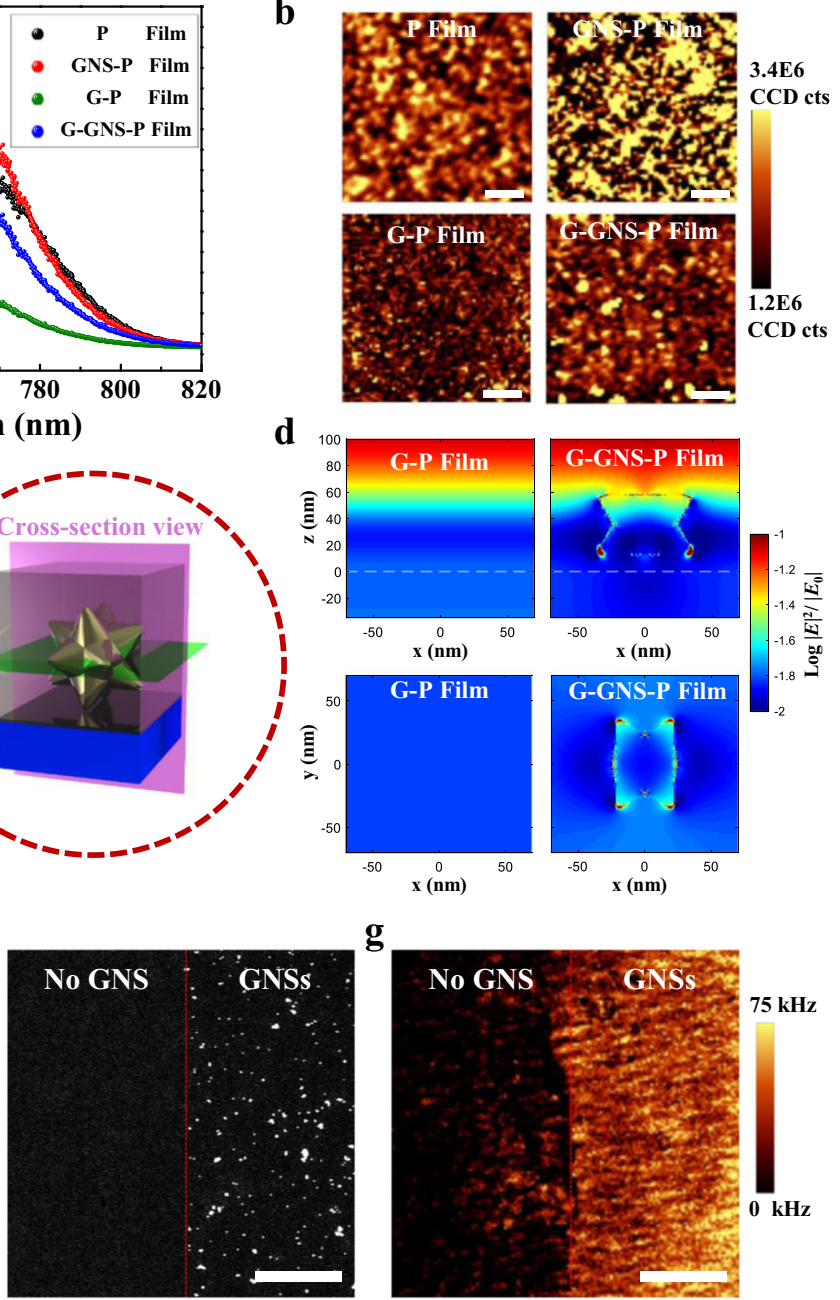

g

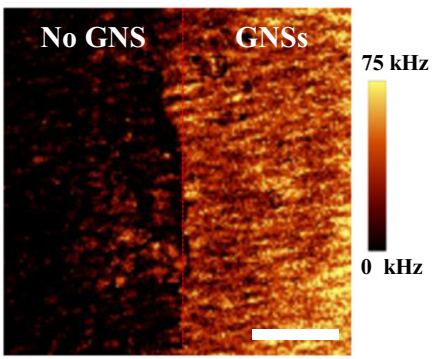

Fig. 5 Optical properties. a PL spectra of the photoactive layers (only-perovskite, GNS-P, G-P, and G-GNS-P) upon excitation at $532 \mathrm{~nm}$. b Integrated PL intensity maps of the photoactive layers (only-perovskite, GNS-P, G-P, and G-GNS-P) upon excitation at $532 \mathrm{~nm}$. A single data point in this map was collected by integrating the local PL signal over wavelengths ranging from 720 to $800 \mathrm{~nm}$ (only-perovskite), 715 to $795 \mathrm{~nm}$ (GNS-P), 710 to $790 \mathrm{~nm}$ (G-P), and 705 to $785 \mathrm{~nm}$ (G-GNS-P) (the scale bar is $10 \mu \mathrm{m}$ ). c FDTD simulation model of the G-GNS-P film on a $\mathrm{SiO}_{2}$ substrate (GNS tip-to-tip distance $=70 \mathrm{~nm}$ ). $\mathbf{d}$ Field intensity patterns calculated from the FDTD model based on the electric responses of the G-P and G-GNS-P films at $460 \mathrm{~nm}$ in cross-sectional view (top) and top view at $z=35 \mathrm{~nm}$ (bottom). e Schematic image of the taping method for fabrication of the GNS selectively coated $\mathrm{Si} / \mathrm{SiO}_{2}$ substrate. $\mathbf{f} \mathrm{SEM}$ (left) and $\mathbf{g} \mathrm{SNOM}$ (right) images of the selectively GNS-coated $\mathrm{Si}_{\mathrm{SiO}}$ substrate (the scale bar is $5 \mu \mathrm{m}$ ).

shown when comparing perovskite-only and GNS-P films without the graphene layer, which indicates the strong resonant coupling between the excitons in the perovskite and the GNS plasmons. Furthermore, the pristine perovskite film exhibited a PL peak at $765 \mathrm{~nm}$ arising from the band gap of the perovskite, while the G-P film showed a slightly blueshifted PL peak. This phenomenon may be due to the low trap density of the perovskite layer of the G-P film, which is cleaner and more pinhole-free than the pristine perovskite layer ${ }^{20,37}$. The introduction of GNSs onto the G-P film also leads to blueshifting of the PL peak, probably due to the plasmonic coupling of GNSs. A previous study also indicated a spectral blueshift with $\mathrm{Au}$ nanoparticles on semiconducting materials corresponding to plasmonic coupling ${ }^{38}$. The local variation in the quenching efficiency within the film was characterized through local integrated PL intensity mapping of the perovskite films (Fig. 5b). The integrated PL mapping images exhibit a tendency consistent with the steady-state PL spectra of the films, showing enhanced fluorescence emission of the perovskite due to the GNS-enhanced plasmonic effect and reduced fluorescence emission of the perovskite due to the efficient charge carrier transfer between the perovskite film and the graphene layer.

To theoretically investigate the scattering and LSPR effects of the GNSs in the PD systems (Fig. 5c), we used a 

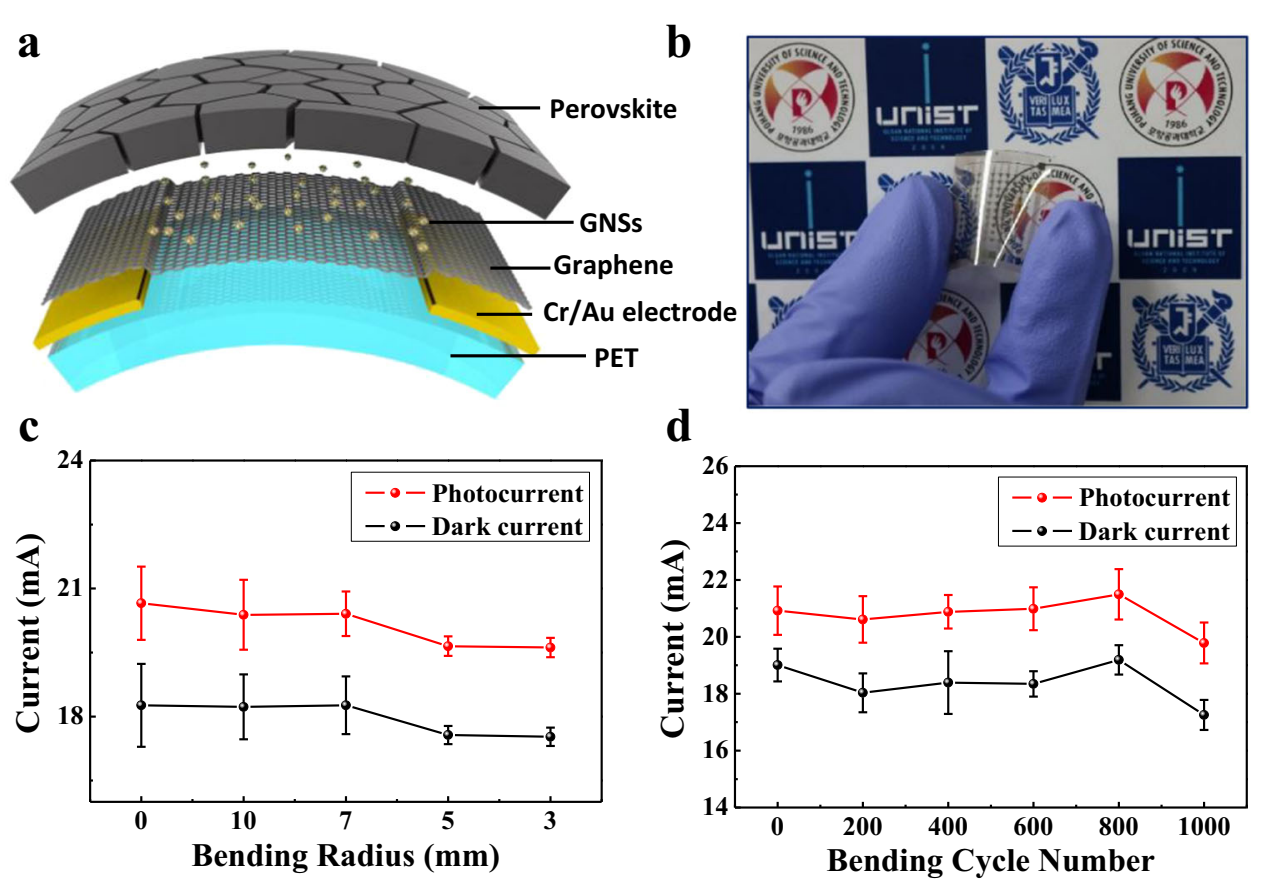

d

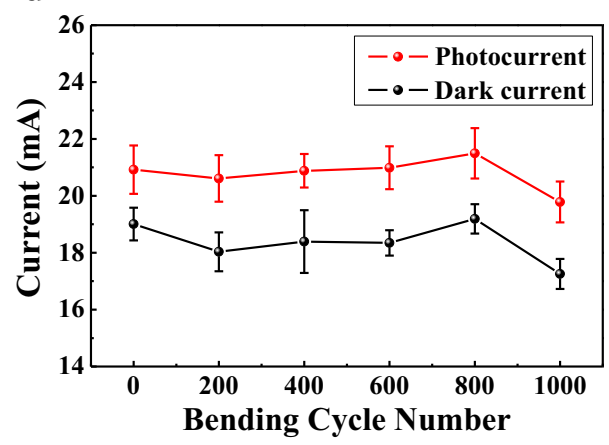

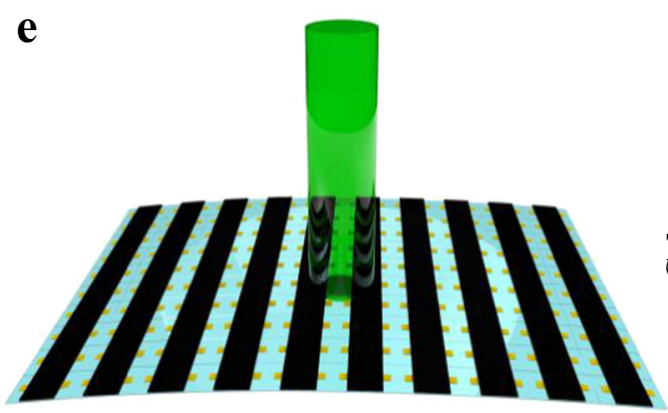

$10 \times 10$ G-GNS-P PD array

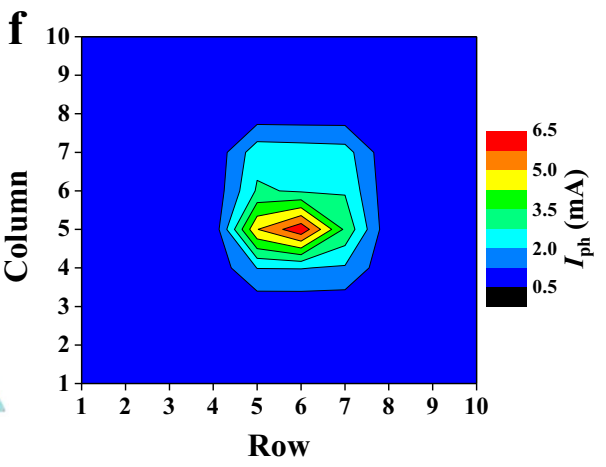

Fig. 6 Evaluation of the flexible photosensor. a Schematic illustration of the flexible G-GNS-P-PD device structure. $\mathbf{b}$ Photograph of the device. Changes in photocurrent and dark current $\mathbf{c}$ under various bending radii (down to $3 \mathrm{~mm}$ ) and $\mathbf{d}$ at various bending cycles (up to $1000 \mathrm{cycles}$ ) under polychromatic light. e Schematic diagram of the $10 \times 10$ G-GNS-P-PD array. $\mathbf{f}$ Photomapping of the G-GNS-P-PD array matrix under light illumination by a green laser $\left(100 \mathrm{~mW} \mathrm{~cm}^{-2}\right)$ positioned at $(6,5)$.

commercial FDTD solver (Lumerical FDTD) to calculate the distribution of the electric field intensity (i.e., $|E(\omega)|^{2} /$ $E_{0}{ }^{2}$ ), transmittance, and reflectance of the system. The optical constant of $\mathrm{Au}$ was taken from tabulated data of Johnson and Christy ${ }^{39}$, and that of the perovskite was retrieved from the ellipsometry measurements in Supplementary Fig. S15. The electric field distributions in the G-P film and G-GNS-P film under blue light were highly intensified around the GNS surface, especially at the perovskite photoactive material (Fig. 5d). The electric field distributions in different types of films under blue, green, and red light are shown in Supplementary Figs. S16-S18, respectively. For a quantitative understanding of the effects of the GNSs, we also investigated the local electric field distributions on GNS-patterned substrates using SNOM. The sample preparation procedure for the SNOM measurement is presented in Fig. 5e. Figure $5 \mathrm{f}$ shows an SEM image of an uncoated (left) and GNScoated (right) substrate. Figure $5 \mathrm{~g}$ shows the corresponding SNOM image of the boundary between the uncoated (left) and GNS-coated (right) areas on the substrate. The SNOM image of the substrate with GNSs clearly shows a higher scattering intensity than that of the uncoated area of the substrate (right side; $532 \mathrm{~nm}$ wavelength). These results indicate that the electric field intensity of the GNS-coated substrate is higher than that of the substrate without GNSs due to the strong plasmonic effects of the GNSs. Overall, the theoretical 
calculation and SNOM analysis confirm that the utilization of GNSs is highly promising for enhancing the electric field via plasmonic effects and for improving the performance of optoelectronic devices.

\section{Performance of flexible photodetector arrays}

To explore the potential applicability as a flexible sensor platform, G-GNS-P-PDs were fabricated on PET substrates (Fig. 6a). Figure 6b shows a photograph of the fabricated G-GNS-P-PDs. Electrical characterization was conducted at various bending radii $\left(R_{\mathrm{B}}\right)$ to assess the mechanical flexibility and operational stability of the PDs. Bending tests were performed at four $R_{B} \mathrm{~s}: 10,7,5$, and $3 \mathrm{~mm}$. Fig. $6 \mathrm{c}$ shows the average $I_{\text {light }}$ and $I_{\text {dark }}$ values of the devices measured at various $R_{B} \mathrm{~S}$, including for the initial and recovered states. Notably, the flexible G-GNSP-PDs exhibited highly stable electrical performances at various $R_{B} \mathrm{~s}$, even as small as $3 \mathrm{~mm}$. The average $I_{\text {light }}$ and $I_{\text {dark }}$ values were monitored for up to 1000 cycles, with an interval of 200 cycles, at an $R_{B}$ of $3 \mathrm{~mm}$ (Fig. 6d). Both factors showed stable retention throughout the repetitive bending tests, indicating that the developed PDs were mechanically and operationally stable. The $R_{B}$ of $3 \mathrm{~mm}$ is four times smaller than the lowest reported $R_{B}(12 \mathrm{~mm})$ of the flexible G-P-PDs in Table S1 (Supplementary Information $)^{23}$. Furthermore, a $10 \times 10$ G-GNS-P-PD matrix was fabricated using the same device configuration and fabrication process as for the unit device. Figure 6e shows schematic and photographic images of the fabricated GGNS-P-PD matrix. The center of the sensing matrix was illuminated by a green laser source, as shown in Supplementary Fig. S19, and the photonic signal was illustrated by two-dimensional mapping as a function of the $R$ value, as shown in Fig. 6f. $R$ values $>6000$ were observed from the unit device only around position $(6,5)$, which corresponds to the illumination spot of the incident laser. This demonstrates the high-performance, flexible, and multifunctional light-detection capability of our G-GNS-P-PDs compared to recently reported G-P-based PDs ${ }^{6,22}$. These results indicate that the G-GNS-P-PDs and their associated fabrication processes can potentially be used to make high-performance flexible optoelectronic devices.

\section{Conclusion}

In conclusion, we fabricated novel flexible graphene dual hybrid PDs with high performance and mechanical robustness by utilizing both perovskite materials and GNSs. The synergistic effects of graphene and the GNSs, which combine high mobility and a strong plasmonic effect, yielded outstanding photodetection performances with high $R$, EQE, and $D^{\prime \prime}$ of $58,952 \mathrm{~A} \mathrm{~W}^{-1}, 1.59 \times 10^{7} \%$, and $1.31 \times 10^{13}$ Jones, respectively. In comparison with the P-PDs, the G-GNS-P-PDs showed greatly enhanced $R$ (by more than 7.7 million-fold) and $D^{*}$ (by more than hundred-fold), whereas the GNS-P-PDs or G-P-PDs exhibited low increments. In addition, flexible G-GNSP-PDs prepared on a plastic substrate showed stable electrical performance and mechanical stability under bending tests with various $R_{B} \mathrm{~S}$ (down to $3 \mathrm{~mm}$ ) and in bending cycle tests $(\approx 1000$ cycles). Moreover, we also confirmed the advantages of our hybrid device systems utilizing graphene and GNSs by using PL mapping, theoretical calculations, and SNOM analysis. Furthermore, the flexible G-GNS-P-PD $10 \times 10$ matrix fabricated on a plastic substrate showed highly sensitive spatiotemporal photomapping behavior with remarkable mechanical durability, demonstrating high potential for use in flexible image sensors. This perovskite-plasmonic nanoparticle hybrid system can be easily applied to a large area and is highly suitable for fabricating high-performance and flexible photosensing systems. These novel flexible graphene hybrid PDs described herein are expected to contribute to the development of imaging sensors suitable for low-light photography, wearable PDs, and UV detectors with high photoresponsivity.

\section{Acknowledgements \\ This work was supported by National Research Foundation of Korea (NRF) grants (Nos. 2020R1A2B5B03094499 and 2018R1A2A1A05079100), the Nano Material Technology Development Program (No. 2017M3A7B8063825), and the Center for Advanced Soft Electronics under the Global Frontier Research Program (2013M3A6A5073175) funded through the NRF by the Ministry of Science and ICT (MSIT), Korea.}

\section{Author details \\ ${ }^{1}$ School of Chemical and Biological Engineering, Institute of Chemical Processes, Seoul National University, 1 Gwanak-ro, Gwanak-gu, Seoul 08826, Republic of Korea. ${ }^{2} S$ chool of Energy and Chemical Engineering, Ulsan National Institute of Science and Technology (UNIST), Ulsan 44919, Republic of Korea. ${ }^{3}$ Thin Film Materials Research Center, Korea Research Institute of Chemical Technology, Post Office Box 107, Yuseong, Daejeon 34114, Republic of Korea. ${ }^{4}$ Department of Chemical Engineering, Pohang University of Science and Technology (POSTECH), Pohang, Gyeongbuk 37673, Republic of Korea. ${ }^{5}$ Department of Mechanical Engineering, Pohang University of Science and Technology (POSTECH), Pohang, Gyeongbuk 37673, Republic of Korea}

\section{Author contributions}

Y.H.L., S.P., H.K., and J.H.O. conceived the idea and designed the research. J.H.O. directed the entire research project. Y.H.L. performed the preparation and characterization of all the samples and devices. H.K. directed the preparation and characterization of GNSs and hybrid films. S.P. and J.Y. performed the preparation and characterization of GNSs and hybrid films. Y.W., J.H.H., J.H.L., S.H.L., and J.P. performed device measurements and analyzed the data. J.R. directed the theoretical study. J.M. performed the DDA simulation. All the authors contributed to the writing of the paper.

Conflict of interest

The authors declare that they have no conflict of interest.

\section{Publisher's note}

Springer Nature remains neutral with regard to jurisdictional claims in published maps and institutional affiliations.

Supplementary information is available for this paper at https://doi.org/ 10.1038/s41427-020-00260-1. 
Received: 7 May 2020 Revised: 21 September 2020 Accepted: 5 October 2020.

Published online: 18 December 2020

\section{References}

1. Mueller, T., Xia, F. \& Avouris, P. Graphene photodetectors for high-speed optical communications. Nat. Photon. 4, 297-301 (2010).

2. Bonaccorso, F., Sun, Z., Hasan, T. \& Ferrari, A. C. Graphene photonics and optoelectronics. Nat. Photon. 4, 611-622 (2010).

3. Romagnoli, M. et al. Graphene-based integrated photonics for nextgeneration datacom and telecom. Nat. Rev. Mater. 3, 392-414 (2018).

4. Bao, Q. \& Loh, K. P. Graphene photonics, plasmonics, and broadband optoelectronic devices. ACS Nano 6, 3677-3694 (2012).

5. Hsieh, Y. -P. et al. Ultra-high sensitivity graphene photosensors. Appl. Phys. Lett. 104, 041110 (2014).

6. Lee, Y. et al. High-performance perovskite-graphene hybrid photodetector. Adv. Mater. 27, 41-46 (2015).

7. Xia, F., Mueller, T., Lin, Y. -M., Valdes-Garcia, A. \& Avouris, P. Ultrafast graphene photodetector. Nat. Nanotechnol. 4, 839-843 (2009).

8. Koppens, F. H. L. et al. Photodetectors based on graphene, other twodimensional materials and hybrid systems. Nat. Nanotechnol. 9, 780-793 (2014).

9. Nair, R. R. et al. Fine structure constant defines visual transparency of graphene. Science 320, 1308-1308 (2008).

10. Fang, Z. et al. Graphene-antenna sandwich photodetector. Nano Lett. 12 3808-3813 (2012)

11. Freitag, M. et al. Photocurrent in graphene harnessed by tunable intrinsic plasmons. Nat. Commun. 4, 1951 (2013).

12. Konstantatos, G. et al. Hybrid graphene-quantum dot phototransistors with ultrahigh gain. Nat. Nanotechnol. 7, 363-368 (2012).

13. Manga, K. K., Wang, S., Jaiswal, M., Bao, Q. \& Loh, K. P. High-gain graphenetitanium oxide photoconductor made from inkjet printable ionic solution. Adv. Mater. 22, 5265-5270 (2010).

14. Huisman, E. H. et al. High gain hybrid graphene-organic semiconductor phototransistors. ACS Appl. Mater. Interfaces 7, 11083-11088 (2015).

15. Yu, H., Joo, P., Lee, D., Kim, B. -S. \& Oh, J. H. Photoinduced charge-carrier dynamics of phototransistors based on perylene diimide/reduced graphene oxide core/shell p-n junction nanowires. Adv. Opt. Mater. 3, 241-247 (2014).

16. Liu, X., Lee, E. K. \& Oh, J. H. Graphene-ruthenium complex hybrid photodetectors with ultrahigh photoresponsivity. Small 10, 3700-3706 (2014).

17. Lee, Y. H., Jang, M., Lee, M. Y., Kweon, O. Y. \& Oh, J. H. Flexible field-effect transistor-type sensors based on conjugated molecules. Chem 3, 724-763 (2017).

18. Lee, Y. H. et al. Recent advances in organic sensors for health self-monitoring systems. J. Mater. Chem. C 6, 8569-8612 (2018).

19. Liu, Y. et al. Plasmon resonance enhanced multicolour photodetection by graphene. Nat. Commun. 2, 579 (2011).
20. Ding, J. et al. High-performance stretchable photodetector based on $\mathrm{CH}_{3} \mathrm{NH}_{3} \mathrm{Pbl}_{3}$ microwires and graphene. Nanoscale 10, 10538-10544 (2018).

21. Wang, Y. et al. Hybrid graphene-perovskite phototransistors with ultrahigh responsivity and gain. Adv. Opt. Mater. 3, 1389-1396 (2015).

22. Sun, Z., Aigouy, L. \& Chen, Z. Plasmonic-enhanced perovskite-graphene hybrid photodetectors. Nanoscale 8, 7377-7383 (2016).

23. Dang, V. Q. et al. Methylammonium lead iodide perovskite-graphene hybrid channels in flexible broadband phototransistors. Carbon 105, 353-361 (2016).

24. Hao, F., Nehl, C. L., Hafner, J. H. \& Nordlander, P. Plasmon resonances of a gold nanostar. Nano Lett. 7, 729-732 (2007).

25. Maiorano, G. et al. Monodispersed and size-controlled multibranched gold nanoparticles with nanoscale tuning of surface morphology. Nanoscale $\mathbf{3}$, 2227-2232 (2011).

26. Haiss, W., Thanh, N. T. K., Aveyard, J. \& Fernig, D. G. Determination of size and concentration of gold nanoparticles from UV-Vis spectra. Anal. Chem. 79, 4215-4221 (2007)

27. Li, X. et al. Large-area synthesis of high-quality and uniform graphene films on copper foils. Science 324, 1312-1314 (2009).

28. Chandrasekhar, P. S. \& Komarala, V. K. Graphene/ZnO nanocomposite as an electron transport layer for perovskite solar cells; the effect of graphene concentration on photovoltaic performance. RSC Adv. 7, 28610-28615 (2017).

29. Zhou, L. et al. Enhanced planar perovskite solar cell efficiency and stability using a perovskite/PCBM heterojunction formed in one step. Nanoscale 10, 3053-3059 (2018).

30. Konstantatos, G., Clifford, J., Levina, L. \& Sargent, E. H. Sensitive solutionprocessed visible-wavelength photodetectors. Nat. Photon. 1, 531-534 (2007).

31. Lee, Y. H. et al. A flexible high-performance photoimaging device based on bioinspired hierarchical multiple-patterned plasmonic nanostructures. Small 14, 1703890 (2018).

32. Fang, Y., Armin, A., Meredith, P. \& Huang, J. Accurate characterization of nextgeneration thin-film photodetectors. Nat. Photon. 13, 1-4 (2019).

33. Rath, A. K. et al. Solution-processed inorganic bulk nano-heterojunctions and their application to solar cells. Nat. Photon. 6, 529-534 (2012).

34. Zhu, Z. et al. Efficiency enhancement of perovskite solar cells through fast electron extraction: the role of graphene quantum dots. J. Am. Chem. Soc. 136, 3760-3763 (2014)

35. Cai, C., Zhai, J., Bi, G. \& Wu, H. Electron energy transfer effect in Au NS/ $\mathrm{CH}_{3} \mathrm{NH}_{3} \mathrm{Pbl}_{3-x} \mathrm{Cl}_{x}$ heterostructures via localized surface plasmon resonance coupling. Opt. Lett. 41, 4297-4300 (2016).

36. Wu, X. et al. Enhancing perovskite film fluorescence by simultaneous nearand far-field effects of gold nanoparticles. RSC Adv. 7, 35752-35756 (2017)

37. Liu, J. et al. Improved crystallization of perovskite films by optimized solvent annealing for high efficiency solar cell. ACS Appl. Mater. Interfaces 7, 24008-24015 (2015).

38. Cai, Y. -Y. et al. Photoluminescence of gold nanorods: purcell effect enhanced emission from hot carriers. ACS Nano 12, 976-985 (2018).

39. Johnson, P. B. \& Christy, R. W. Optical constants of the noble metals. Phys. Rev. B 6, 4370-4379 (1972). 